\title{
Shape evolution in yttrium and niobium neutron-rich isotopes
}

\author{
R. Rodriguez-Guzman, ${ }^{1}$ P. Sarriguren, ${ }^{1}$ and L. M. Robledo ${ }^{2}$ \\ ${ }^{1}$ Instituto de Estructura de la Materia, Consejo Superior de Investigaciones Científicas, Serrano 123, E-28006 Madrid, Spain \\ ${ }^{2}$ Departamento de Física Teórica, Módulo 15, Universidad Autónoma de Madrid, E-28049 Madrid, Spain
}

(Received 16 February 2011; published 11 April 2011)

\begin{abstract}
The isotopic evolution of the ground-state nuclear shapes and the systematics of one-quasiproton configurations are studied in neutron-rich odd- $A$ yttrium and niobium isotopes. We use a self-consistent Hartree-FockBogoliubov formalism based on the Gogny energy density functional with two parametrizations, D1S and $\mathrm{D} 1 \mathrm{M}$. The equal-filling approximation is used to describe odd- $A$ nuclei preserving both axial and time-reversal symmetries. Shape-transition signatures are identified in the $N=60$ isotopes in both the charge radii and spin parities of the ground states. These signatures are a common characteristic for nuclei in the whole mass region. The nuclear deformation and shape coexistence inherent to this mass region are shown to play a relevant role in the understanding of the spectroscopic features of the ground and low-lying one-quasiproton states. Finally, a global picture of the neutron-rich $A \sim 100$ mass region from krypton up to molybdenum isotopes is illustrated with the systematics of the nuclear charge radii isotopic shifts.
\end{abstract}

DOI: 10.1103/PhysRevC.83.044307

PACS number(s): 21.60.Jz, 21.10.Pc, 27.60.+j

\section{INTRODUCTION}

Structural evolution as a function of the number of nucleons is a subject of increasing interest in nuclear structure, which is supported by very intense activity on both the theoretical and experimental sides [1-13]. In particular, neutron-rich nuclei in the mass region around $A=100$ have received special attention because of the interesting features of nuclear structure that merge there [8,14-17].

Experimentally, the efforts are focused on different and complementary directions. The most relevant for the purpose of this work are related, on the one hand, to the mass determination [9-11] in the case of exotic neutron-rich nuclei and to the accuracy required for the modeling of astrophysical events $[18,19]$. In particular, the mass region we are concerned with here is highly significant to understanding the nucleosynthesis path and the isotopic abundances generated by the astrophysical r-process [20]. On the other hand, in laser spectroscopy experiments the focus is aimed at measuring nuclear spins, magnetic dipole moments, spectroscopic quadrupole moments, and mean-square charge radii from isotopic shifts. Considerable progress has been achieved in recent years (for a review, see [8] and references therein), and the mass region studied in this work has received special attention [13,21-36].

The region has been studied theoretically using phenomenological models [37-40] and microscopic approaches based on the relativistic mean field (RMF) [41], as well as nonrelativistic Skyrme [42,43] and Gogny [14-16,44,45] energy density functionals (EDFs). All in all, the accumulated information on this mass region has established some characteristic features that can be associated with signatures of a shape transition at $N=60$.

Different nuclear properties sensitive to these structural changes have been recently investigated [14-16] in several isotopic chains in this mass region. We used a self-consistent Hartree-Fock-Bogoliubov (HFB) approximation based on the finite range and density-dependent Gogny EDF [46] and the equal-filling approximation (EFA) to deal with the odd nucleon. We analyzed bulk and spectroscopic properties of neutron-rich isotopes with both even- $Z$ ( $\mathrm{Sr}, \mathrm{Zr}$, and $\mathrm{Mo}$ ) isotopes and odd- $Z(\mathrm{Rb})$ isotopes. Our purpose in this paper is to complete the systematic study of the bulk and spectroscopic properties in two chains of odd- $Z$ isotopes, yttrium $(Z=39)$ and niobium $(Z=41)$, as well as in the chain of krypton isotopes $(Z=36)$.

The description of odd- $A$ nuclei involves additional difficulties because the exact blocking procedure requires the breaking of time-reversal invariance, making the calculations more involved [47-51]. In the present study, we use the EFA, a prescription widely used in mean-field calculations to preserve the advantages of time-reversal invariance. The predictions arising from various treatments of the blocking have been studied in Ref. [50], which concluded that the EFA is sufficiently precise for most practical applications. More details of our procedure can be found in Refs. [15,49].

In this work we consider two parametrizations of the Gogny EDF, namely, D1S [52], as the standard and most studied parameter set [44,45,53-55], and D1M [56], as the most recent effort to find a parametrization that improves the predictions for masses while maintaining the excellent performance and predictive power of the former D1S. Our aim is, first, to verify the robustness of our predictions with respect to the particular version of the EDF employed and, second, to test the performance of D1M in the present context of the spectroscopy of odd- $A$ nuclei.

The paper is organized as follows. In Sec. II, we present a brief description of the theoretical formalism used in the present work (i.e., the HFB-EFA framework). The results of our calculations for the considered nuclei are discussed in Sec. III, where we pay attention to the one-quasiparticle states and their spectroscopic evolution along the $\mathrm{Y}$ and $\mathrm{Nb}$ isotopic chains. We also compare our results with the available experimental data for charge radii and two-neutron separation energies. In Sec. IV we show the results for krypton isotopes, as well as the systematics of the charge radii in the whole 
region from krypton $(Z=36)$ up to molybdenum $(Z=42)$. Finally, Sec. V is devoted to concluding remarks and work perspectives.

\section{THEORETICAL FRAMEWORK}

Our theoretical framework to deal with odd- $A$ nuclei is based on the Gogny-HFB-EFA formalism. In previous studies of even-even nuclei [6,7] we have found the so-called gradient method [57] advantageous to obtain the solution of the HFB equations, leading to the (even number parity) vacuum $|\Phi\rangle$. In this method, the HFB equation is recast in terms of a minimization (variational) process of the mean-field energy. The Thouless parameters defining the most general HFB wave functions [58] are used as variational parameters. As is customary in calculations with the Gogny force, the kinetic energy of the center-of-mass motion has been subtracted from the Routhian to be minimized in order to ensure that the center of mass is kept at rest. The exchange Coulomb energy was considered in the Slater approximation and the contribution of the Coulomb interaction to the pairing field is neglected. Both axial and time-reversal symmetries are self-consistent in our calculations for odd- $A$ nuclei. Triaxial calculations have also been performed in the case of even-even $\mathrm{Kr}$ isotopes.

The HFB ground-state wave function $|\Phi\rangle$ of an even-even nucleus is defined by the condition of being the vacuum of the annihilation quasiparticle operators $\beta_{\mu}$ of the Bogoliubov transformation [58,59]. On the other hand, the ground and low-lying one-quasiparticle states of odd- $A$ systems, like the ones considered in the present work, can be handled with blocked (odd number parity) [58,59] HFB wave functions

$$
\left|\Psi_{\mu_{B}}\right\rangle=\beta_{\mu_{B}}^{+}|\Phi\rangle
$$

where $\mu_{B}$ indicates the quasiparticle state to be blocked and stands for the indexes compatible with the symmetries of the odd- $A$ nuclei, such as the angular momentum projection $K$ and parity $\pi$ in the case of axial symmetry. As mentioned above, here we use the EFA to the exact blocking that preserves time-reversal invariance. In this approximation the unpaired nucleon is treated on an equal footing with its time-reversed state by sitting half a nucleon in a given orbital and the other half in its time-reversed partner. The microscopic justification has been given in Ref. [49] using ideas of quantum statistical mechanics. The EFA energy can be obtained as the statistical average, with a given density matrix operator, and, by applying the variational principle to it, the HFB-EFA equation [49] is obtained. The existence of a variational principle allows the use of the gradient method to solve the HFB-EFA equation with the subsequent simplification in the treatment of the constraints.

The solution of the HFB-EFA (as well as the exact blocked HFB) equation depends upon the initial blocked level $\mu_{B}$. In the HFB-EFA case, the $K$ quantum number is self-consistently preserved along the calculation, as is the parity if octupole correlations are not allowed in the iterative process. Blocking levels with different $K^{\pi}$ values lead to different quantum states of the odd- $A$ nucleus, the ground state being the one with the lowest energy. One should note that because of the self-consistent nature of the whole procedure, for a given $K^{\pi}$ there is no guarantee that the lowest-energy solution for those $K^{\pi}$ values is obtained by taking the quasiparticle with the lowest energy as the initial blocked state. Therefore, several quasiparticles with the same $K^{\pi}$ must be considered. In addition, in the present case and because of the presence of coexisting prolate, oblate, and spherical minima in some of the nuclei considered, blocked configurations with those quadrupole deformations have to be explored. Constrained calculations have been performed to generate potential energy curves (PECs) for the even-even neighbor nuclei. One can find a systematic compilation of PECs obtained with Gogny D1S in Ref. [60]. In the case of odd- $A$ nuclei, the purpose of the computation of such PECs is twofold: first, they give us initial hints on the evolution of the different competing shapes in the considered nuclei and, second, they provide a whole set of prolate, spherical, and oblate even-even HFB states (reference states) for the subsequent treatment of the neighboring odd- $A$ nuclei. In fact, once a reference (even-even) state with a given deformation is chosen, we use it to perform an additional (constrained) HFB calculation providing an unblocked fully paired state corresponding to an odd average neutron number (false vacuum [47]) with the same deformation. Such prolate, spherical, and oblate false vacua are then used as input configurations in our subsequent blocking scheme (i.e., EFA).

Thus, the minimization process has to be carried out several times, using different initial prolate, spherical, and oblate (false) vacua. We have repeated each calculation, for a given false vacuum and $K$ values from $1 / 2$ up to $15 / 2$, using as initial blocking states the 12 quasiparticles corresponding to the lowest quasiparticle energies. The use of so many initial configurations guarantees that we are not missing the true ground state and all the lowest excited states. Note that for nuclei in this region of the nuclear chart, there exist several competing shapes at low excitation energy and therefore our procedure ensures that the lowest-energy solution can be reached for all values of the quadrupole moment $Q_{20}$ and mass number.

\section{RESULTS FOR ODD-A YTTRIUM AND NIOBIUM ISOTOPES}

Being odd- $Z$ nuclei, the spin and parity of odd- $A$ yttrium and niobium isotopes are determined by the state occupied by the unpaired proton. The spectroscopic properties of the odd- $A$ isotopes are determined by the one-quasiproton configurations that, in principle, are expected to be rather stable against variations in the number of even neutrons. However, as is known for neighboring nuclei, the isotopes approaching $N \sim 60$ become well deformed $[1,14]$ and the abrupt change in deformation induces signatures in nuclear bulk properties like the two-neutron separation energies and the nuclear charge radii, as well as in spectroscopic properties. In particular, the spin and parity of the nuclear ground state might flip suddenly from one isotope to another, reflecting the structural change.

\section{A. Low-lying one-quasiparticle states}

In Fig. 1 we can see the experimental excitation energies and spin-parity assignments [Fig. 1(a)] in odd- $A$ neutron-rich yttrium isotopes [61]. They are compared to the 

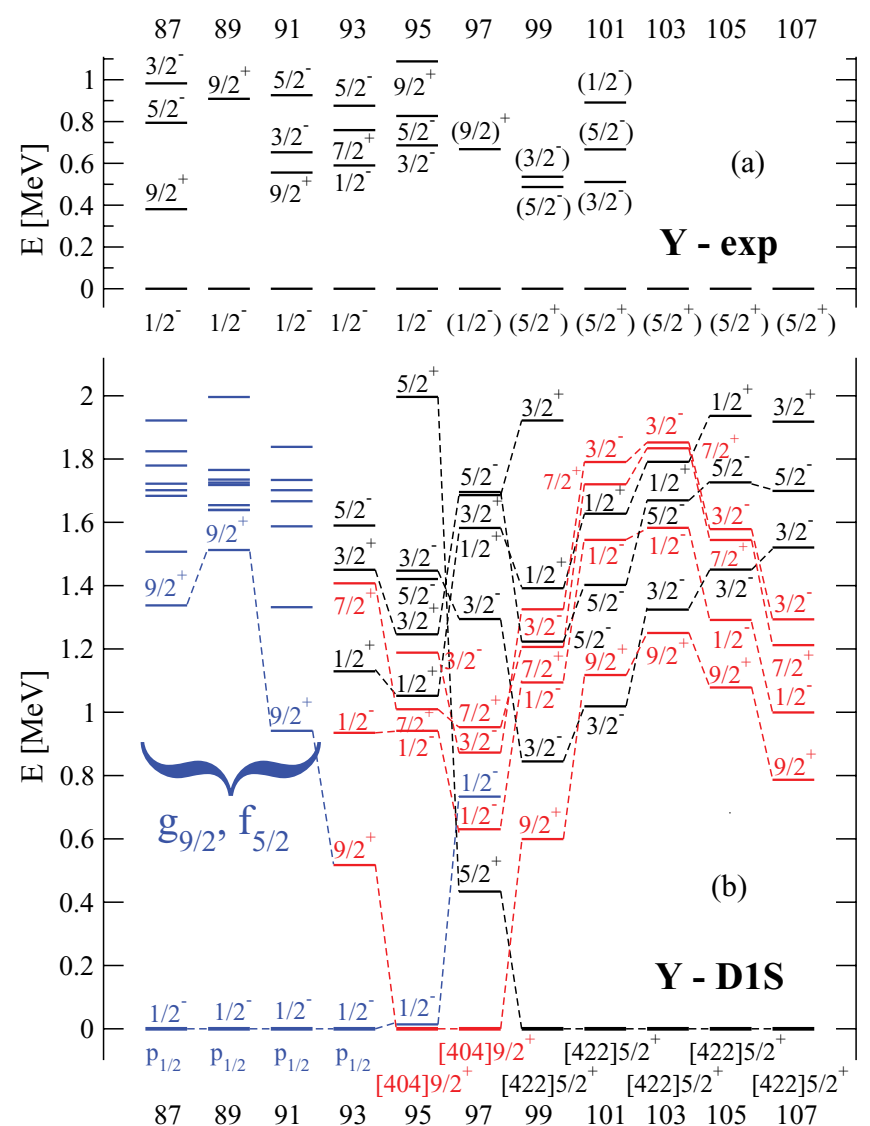

A

FIG. 1. (Color online) Experimental (a) excitation energies and spin-parity assignments of the noncollective states compared with HFB-EFA results (b) for the one-quasiproton states in odd- $A$ yttrium isotopes. Prolate configurations are shown by black lines, oblate ones by red lines, and spherical ones by blue lines.

one-quasiproton states predicted by our Gogny-D1S HFB-EFA calculation [Fig. 1(b)]. The excited states for a given isotope are referred to the corresponding ground state, regardless of its shape. Prolate configurations in our calculations are shown by black lines, oblate ones by red lines, and spherical ones by blue lines. The quasiparticle states are labeled by their $K^{\pi}$ quantum numbers. The most important configurations are joined by dashed lines following the isotopic evolution. In addition, the ground states are labeled by their asymptotic quantum numbers $\left[N, n_{z}, \Lambda\right] K^{\pi}$.

Experimentally, we observe $J^{\pi}=1 / 2^{-}$ground states in $87,89,91,93,95,97 \mathrm{Y}$, then we have $\left(5 / 2^{+}\right)$states in the heavier isotopes $99,101,103,105,107 \mathrm{Y}$, although their assignments are uncertain in these cases. The $9 / 2^{+}$states appear as excited states in the lighter isotopes, while $3 / 2^{-}$and $5 / 2^{-}$excited states are also found in most isotopes, but especially in the heavier ones. The most striking feature observed is the abrupt transition at $N=60(A=99)$ from $\left(1 / 2^{-}\right)$to $\left(5 / 2^{+}\right)$ground states.

The theoretical interpretation of these findings can be understood from the analysis of our results in the lower panel 1(b). According to our calculations, yttrium isotopes evolve from spherical shapes in ${ }^{87-93} \mathrm{Y}$ around $N=50$, with the spherical $p_{1 / 2}$ shell in the ground state and close $g_{9 / 2}$ and $f_{5 / 2}$ as excited states, to slightly deformed oblate shapes in ${ }^{95,97} \mathrm{Y}$, and finally to well-deformed prolate shapes in ${ }^{99-107} \mathrm{Y}$. In the lighter isotopes the ground states correspond to $1 / 2^{-}$ states $\left(p_{1 / 2}\right)$, whereas the excited states correspond to the split $K^{\pi}$ levels coming from $g_{9 / 2}$ and $f_{5 / 2}$, with $9 / 2^{+}$states as the lowest excited states, in agreement with the measured low-lying spectra. The excited $9 / 2^{+}$states decrease in energy as we move away from the magic neutron number $N=50$ because of the incipient oblate deformation emerging, and they eventually become the ground state in ${ }^{97} \mathrm{Y}$. In the case of the ${ }^{95} \mathrm{Y}$ isotope the oblate $9 / 2^{+}$state is practically degenerate with the $1 / 2^{-}$spherical state. In our description, the nucleus ${ }^{97} \mathrm{Y}$ displays a $9 / 2^{+}$oblate ground state with a $1 / 2^{-}$excited state at $0.7 \mathrm{MeV}$ and an incipient prolate $5 / 2^{+}$at $0.4 \mathrm{MeV}$ that will become the ground state in the heavier isotopes ${ }^{99-107} \mathrm{Y}$. All of these heavier prolate isotopes present oblate $9 / 2^{+}$excited states at energies in the range $0.6-1.2 \mathrm{MeV}$. Thus, the leap of the spin-parity of the ground states observed at $N=60$ is well accounted for by the present calculations, which is interpreted as a sudden shape change of the ground state.

Similar to Figs. 1 and 2 shows the corresponding results for niobium isotopes. Experimentally [61] we observe $J^{\pi}=9 / 2^{+}$ ground states in the lighter ${ }^{89-99} \mathrm{Nb}$ isotopes with $1 / 2^{-}$excited
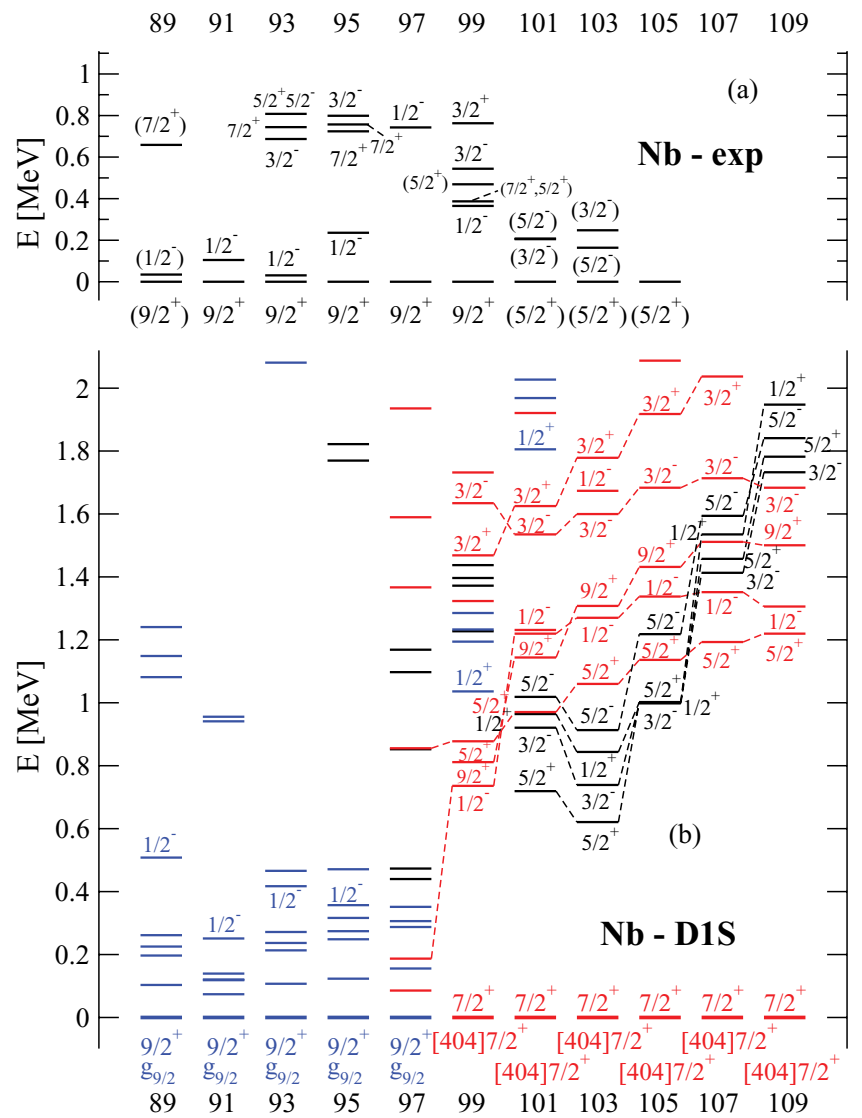

A

FIG. 2. (Color online) Same as in Fig. 1, but for niobium isotopes. 
states at close energy, then we find $\left(5 / 2^{+}\right)$ground states in the heavier isotopes ${ }^{101-105} \mathrm{Nb}$, although the spin-parity assignments are still uncertain in these isotopes, as in the case of the $\mathrm{Y}$ isotopes. The characteristic jump at $N=60(A=101)$ from $9 / 2^{+}$to $\left(5 / 2^{+}\right)$ground states is found once again. The calculations from Gogny D1S [Fig. 2(b)] show a clear correspondence in the lighter isotopes between the measured ground state $J^{\pi}=9 / 2^{+}$and the calculated ones. The observed excited states $1 / 2^{-}$are also labeled in the calculations. They correspond to the excited states from the $p_{1 / 2}$ shell. However, at variance with experiment, our results indicate a transition in ${ }^{99} \mathrm{Nb}$ to the $7 / 2^{+}$oblate state. This oblate configuration is kept all the way up to the heaviest isotope considered, ${ }^{109} \mathrm{Nb}$. The observed $\left(5 / 2^{+}\right)$ground states are found in our calculations as prolate configurations at excitation energies between 0.6 and $1 \mathrm{MeV}$ in ${ }^{101,103,105} \mathrm{Nb}$. It will be interesting to see whether the experimental ground states of ${ }^{101,103,105} \mathrm{Nb}$ are confirmed to be $5 / 2^{+}$states and to measure the heavier ${ }^{107,109} \mathrm{Nb}$, where, according to the calculations, the $5 / 2^{+}$states appear at a very high excitation energy relative to the ground state.

The disagreement between the theoretical predictions and experimental data for the spin and parity of the heavier $\mathrm{Nb}$ isotopes can be understood if triaxiality effects are invoked. In Fig. 3, where the proton single-particle levels for ${ }^{100} \mathrm{Zr}$ are depicted as functions of the deformation parameter $\beta$ [4], we observe for $\beta=-0.2$ (the position of the oblate minimum) a $K^{\pi}=7 / 2^{+}$orbital just above the Fermi level. The occupancy of this orbital produces the $7 / 2^{+}$oblate ground state in ${ }^{101} \mathrm{Nb}$. The $K^{\pi}=5 / 2^{+}$orbital that comes from the same $g_{9 / 2}$ subshell and is presumably responsible for the experimental spin and parity lies higher in energy. The situation is reversed in the prolate side where at $\beta=0.35$ the $K^{\pi}=5 / 2^{+}$orbital is below

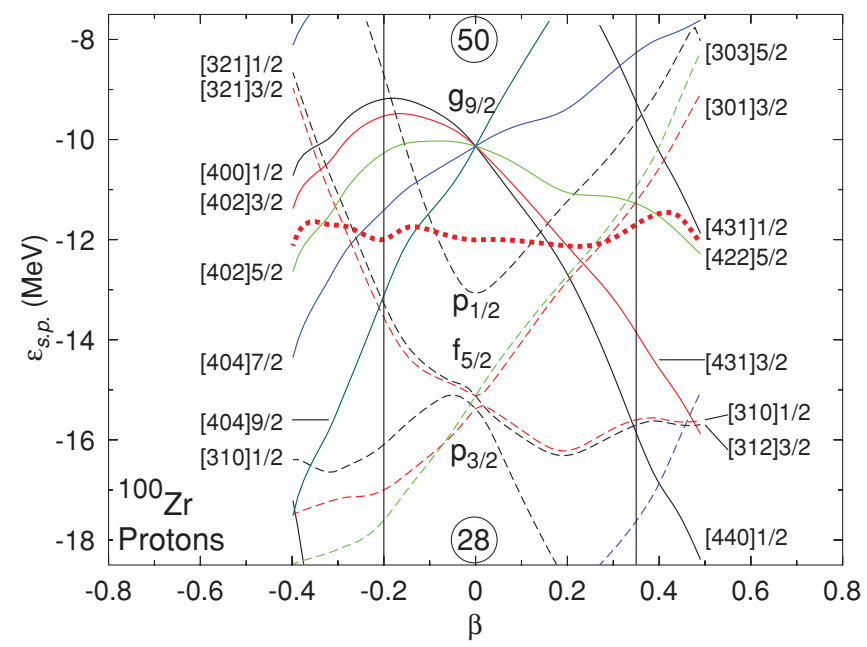

FIG. 3. (Color online) Proton single-particle energies for ${ }^{100} \mathrm{Zr}$ as a function of the quadrupole deformation parameter $\beta$. The Fermi level is plotted by a thick dotted line. The results correspond to the Gogny-D1S EDF. Solid lines correspond to levels with positive parity, whereas dashed lines correspond to negative-parity states. Asymptotic Nilsson quantum numbers $\left[N, n_{z}, \Lambda\right] K^{\pi}$ are also shown at the vertical lines where the minima of the potential energy curves are located. the $K^{\pi}=7 / 2^{+}$one and is still above the Fermi level. The easiest way to connect the oblate and prolate sides is through the triaxial $\gamma$ degree of freedom, with $\gamma$ ranging from $60^{\circ}$ (oblate side) to $0^{\circ}$ (prolate side). It is obvious that the energy of the $K^{\pi}=5 / 2^{+}$orbital must go below the energy of the $K^{\pi}=7 / 2^{+}$orbital at some $\gamma$ value and it is very likely that at that point the blocking of the $K^{\pi}=5 / 2^{+}$orbital will produce the ground-state minimum. This is obviously a hand-waving argument as the $K$ quantum number is not preserved along the $\gamma$ path but, again, it is very likely that both components $K^{\pi}=5 / 2^{+}$and $K^{\pi}=7 / 2^{+}$are going to be dominant in the orbital just above the Fermi level. The above argument calls for the necessity of carrying out a full triaxial calculation for the heaviest odd- $Z \mathrm{Nb}$ isotopes, which is, for the moment, not possible as it will require an extension of our present computational codes for odd nuclei to include the role of triaxiality. Work along these lines is in progress.

In the next figures we compare the spectroscopic properties of the Gogny-D1S and Gogny-D1M parametrizations. In Figs. 4-7 we have separated the prolate (a) and oblate (b) states and have plotted the most relevant hole states below zero energy and the particle states above. The ground states, either oblate or prolate, are indicated with a circle. Specifically, Figs. 4 and 5 correspond to Y isotopes with Gogny D1S and
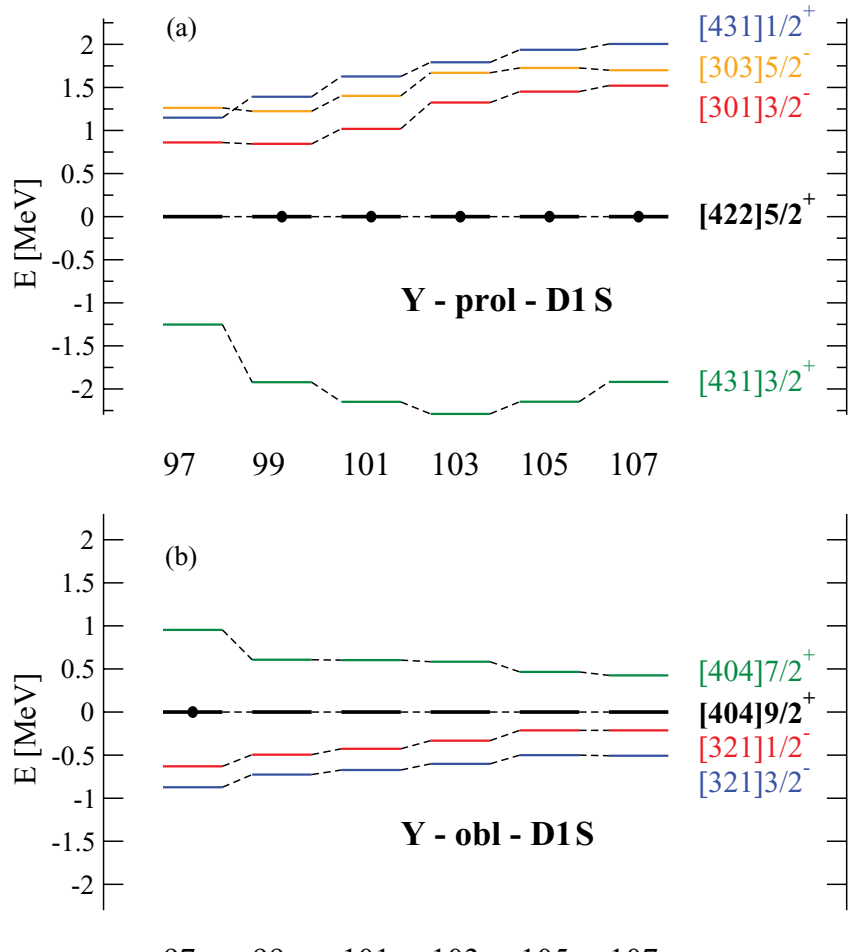

$\begin{array}{llllll}97 & 99 & 101 & 103 & 105 & 107\end{array}$

A

FIG. 4. (Color online) Gogny-D1S excitation energies of singlequasiproton prolate (a) and oblate (b) states in yttrium isotopes. Hole states are plotted below zero energy and particle states are plotted above. The absolute ground states are indicated with a circle. 

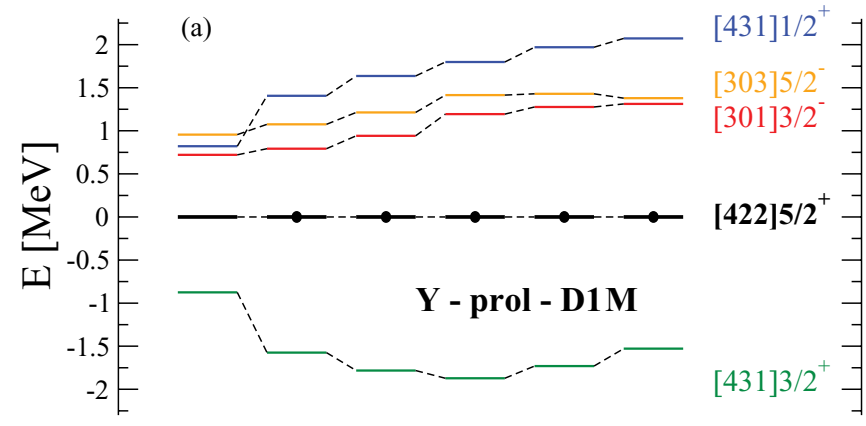

$\begin{array}{llllll}97 & 99 & 101 & 103 & 105 & 107\end{array}$

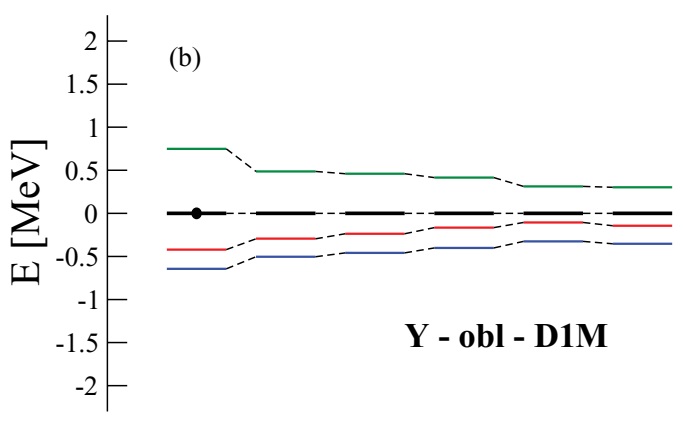

$\begin{array}{llllll}97 & 99 & 101 & 103 & 105 & 107\end{array}$

A

FIG. 5. (Color online) Same as in Fig. 4, but for Gogny D1M.

Gogny D1M, respectively, whereas Figs. 6 and 7 correspond to $\mathrm{Nb}$ isotopes with Gogny D1S and Gogny D1M, respectively.

In Figs. 4 and 5 for yttrium, in the prolate case (a) we have depicted the evolution of the $5 / 2^{+}\left(g_{9 / 2}\right)$ states, which are ground states for ${ }^{99-107} \mathrm{Y}$. In the upper region we find the $3 / 2^{-}\left(f_{5 / 2}\right), 5 / 2^{-}\left(f_{5 / 2}\right)$, and $1 / 2^{+}\left(d_{5 / 2}\right)$ states, while in the lower region we have the $3 / 2^{+}\left(g_{9 / 2}\right)$ state, as shown in Fig. 3 on the prolate side. Similar states are found in the prolate graphs (a) of Figs. 6 and 7 for Nb isotopes, but in this case the prolate configurations are not ground states. In the oblate case (b) we can see for $\mathrm{Y}$ isotopes the $9 / 2^{+}\left(g_{9 / 2}\right)$ state, which is ground state in ${ }^{97} \mathrm{Y}$, the $7 / 2^{+}\left(g_{9 / 2}\right)$ state as a particle state, and the $1 / 2^{-}$and $3 / 2^{-}$from $f_{5 / 2}$ as hole states, as shown in Fig. 3 on the oblate side. The oblate configurations in $\mathrm{Nb}$ isotopes show the $7 / 2^{+}\left(g_{9 / 2}\right)$ as the ground state in all the isotopes depicted. In addition to the states shown for $\mathrm{Y}$ isotopes, here we also show the particle states $5 / 2^{+}$and $3 / 2^{+}$from $g_{9 / 2}$.

Very similar results are obtained from both parametrizations of the Gogny EDF. The only difference worth mentioning is that D1M produces slightly lower excited states and then a more compact level density. This feature can be understood from its larger effective mass that makes the single-particle spectrum somewhat more dense with D1M. This answers our original question about the robustness of the calculations and the reliability of D1M with regard to the spectroscopic properties of the two parametrizations.

To further illustrate the role of deformation and spin-parity assignments in the isotopic evolution, in Fig. 8 we display the
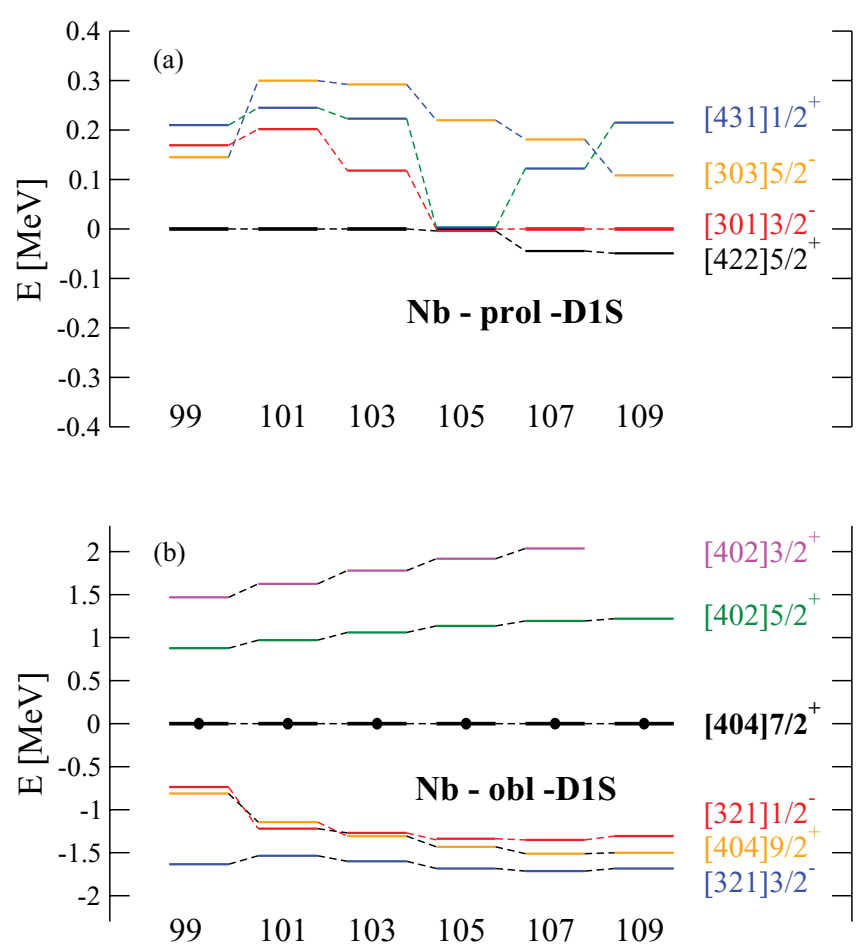

A

FIG. 6. (Color online) Same as in Fig. 4, but for niobium isotopes.

axial quadrupole deformation $\beta$ of the energy minima as a function of $N$ with both D1S and D1M parametrizations for $\mathrm{Y}$ [Fig. 8(a)] and $\mathrm{Nb}$ [Fig. 8(b)] isotopes. The deformation of the ground state for each isotope is circled. We can see that beyond the semi-magic isotope with $N=50$ we start getting two minima in the prolate and oblate sectors. In the case of $\mathrm{Y}$ isotopes, the spherical $1 / 2^{-}$states are ground states up to $N=54$, in agreement with experiment. For $N=52$ and $N=54$ one can see the appearance of an oblate $9 / 2^{+}$state and a prolate $1 / 2^{+}$excited state, which are almost degenerate with the ground state. The next isotopes, with $N=56$ and $N=58$, display a slightly oblate $9 / 2^{+}$ground state and then suddenly a transition occurs at $N=60$ to well-deformed prolate $5 / 2^{+}$ground states, in agreement with the experiment. The calculations with both D1S and D1M are coincident. On the other hand, in the case of $\mathrm{Nb}$ isotopes, we observe the spherical $9 / 2^{+}$states being ground states along $N=44-56$ in agreement with the experiment. However, starting at $N=56$ in the case of D1M and at $N=58$ in the case of D1S, oblate $7 / 2^{+}$ ground states are obtained at variance with the experimental assignment $\left(5 / 2^{+}\right)$for these states, which is nevertheless still uncertain, as mentioned above.

A qualitative understanding of the features just discussed can be obtained by looking at the single-particle levels for protons in ${ }^{100} \mathrm{Zr}$ and depicted in Fig. 3 as a function of the $\beta$ deformation parameter. This $Z=40$ even-even nucleus is in between $\mathrm{Y}$ and $\mathrm{Nb}$ with regard to the number of protons and therefore its single-particle properties should not differ very much (at least at a qualitative level) from those of $\mathrm{Y}$ and 

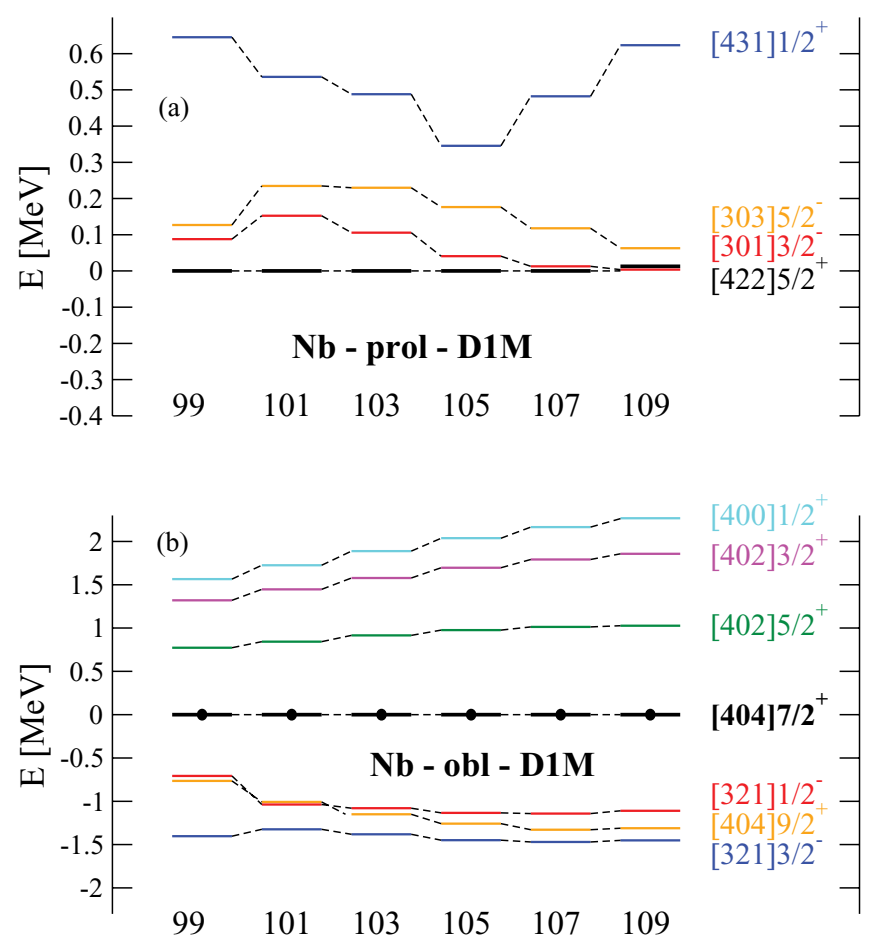

A

FIG. 7. (Color online) Same as in Fig. 5, but for niobium isotopes.
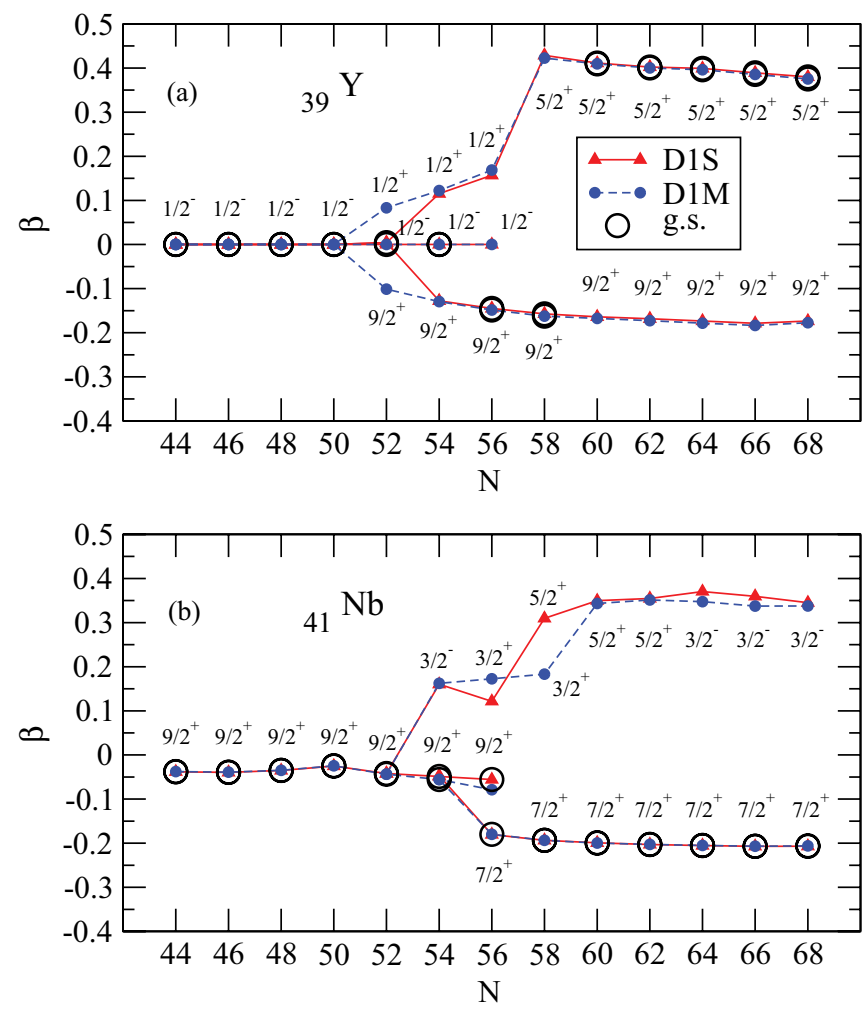

FIG. 8. (Color online) Isotopic evolution of the quadrupole deformation parameter $\beta$ of the energy minima obtained from Gogny D1S and D1M calculations for yttrium (a) and niobium (b) isotopes.
$\mathrm{Nb}$. The insight obtained from Fig. 3 can only be qualitative owing to the self-consistent character of the EFA that somehow incorporates the polarization effects induced by the single proton in the even-even core.

\section{B. Charge radii and two-neutron separation energies}

In Figs. 9-12, we show the results for the charge radii differences (a) defined as $\delta\left\langle r_{c}^{2}\right\rangle^{50, N}=\left\langle r_{c}^{2}\right\rangle^{N}-\left\langle r_{c}^{2}\right\rangle^{50}$, calculated with the same corrections as in Ref. [14], and for the two-neutron separation energies $S_{2 n}$ (b). Figures 9 and 10 show the results for yttrium isotopes calculated with Gogny D1S and Gogny D1M, respectively. Figures 11 and 12 show the corresponding results for niobium isotopes. Our results are compared with isotope shifts from laser spectroscopy experiments $[30,33,36]$ in the case of $\delta\left\langle r_{c}^{2}\right\rangle$ and with mass measurements from Refs. [31,62] in the case of $S_{2 n}$. We plot the results corresponding to the spherical, oblate, and prolate shapes at the minima of the PECs. Results corresponding to the ground states are circled in each isotope.

The first thing to notice is the remarkable similarity between the results obtained with Gogny D1S and Gogny D1M. The only visible difference between D1S and D1M is a somewhat better agreement with the experimental $S_{2 n}$ values in the case of D1M, as can be expected from its improved fitting protocol, which focuses on a more accurate reproduction of the nuclear masses.

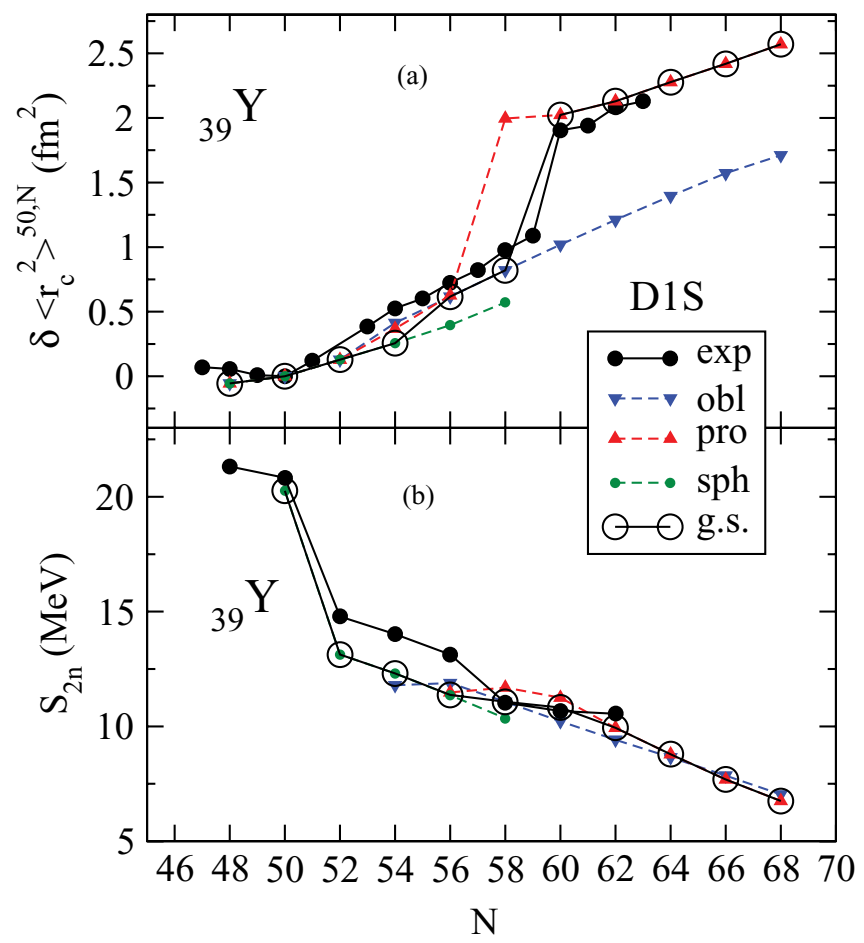

FIG. 9. (Color online) Gogny-D1S-HFB results for $\delta\left\langle r_{c}^{2}\right\rangle$ (a) and $S_{2 n}$ (b) in odd- $A$ yttrium isotopes compared to experimental data from Refs. [31,62] for masses and from Refs. [30,36] for radii. Results for prolate, oblate, and spherical minima are displayed with different symbols (see legend). Open circles correspond to ground-state results. 


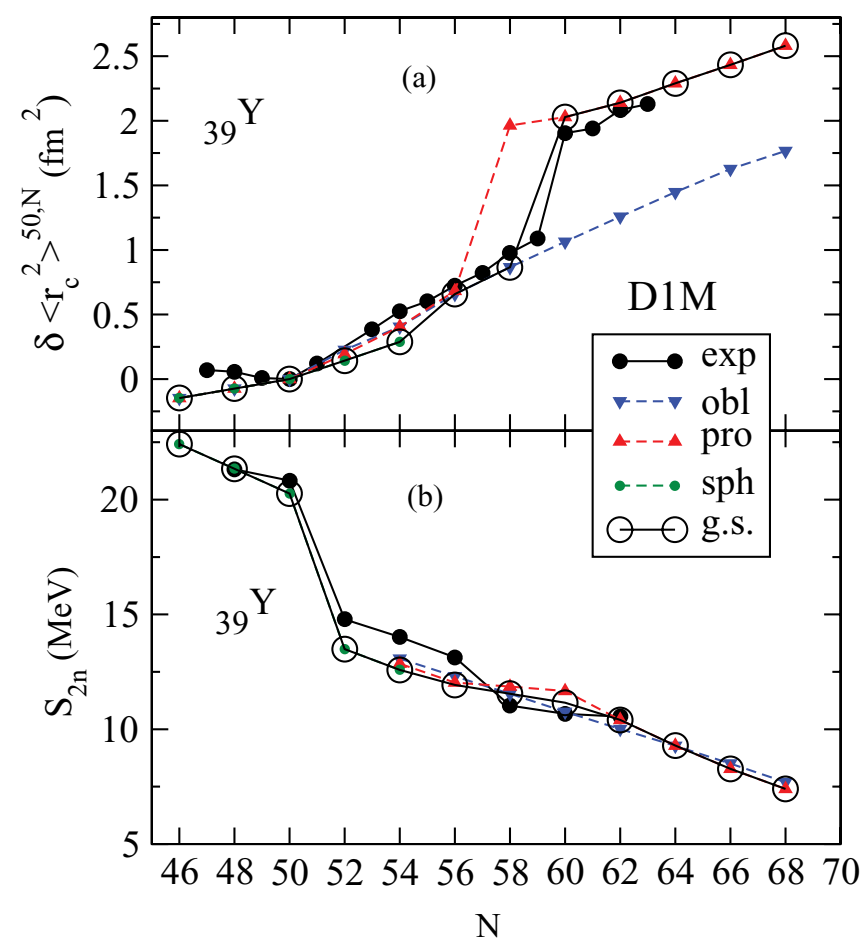

FIG. 10. (Color online) Same as in Fig. 9, but for Gogny D1M.

For $\mathrm{Y}$ isotopes, the measured nuclear charge radii differences depicted in (a) exhibit a sizable jump at $N=60$ where the radius suddenly increases. This observation is well accounted for in our calculations, where the circled ground states show that a jump of the same magnitude occurs between

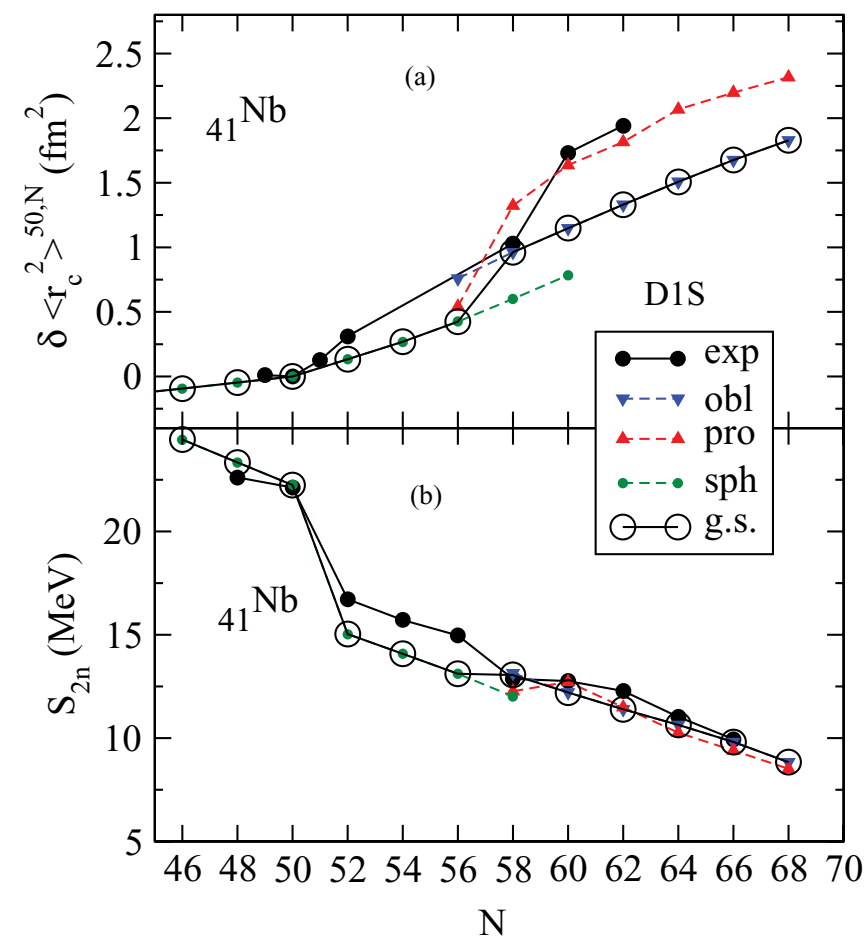

FIG. 11. (Color online) Same as in Fig. 9, but for niobium isotopes. Experimental data for radii are from Ref. [33].

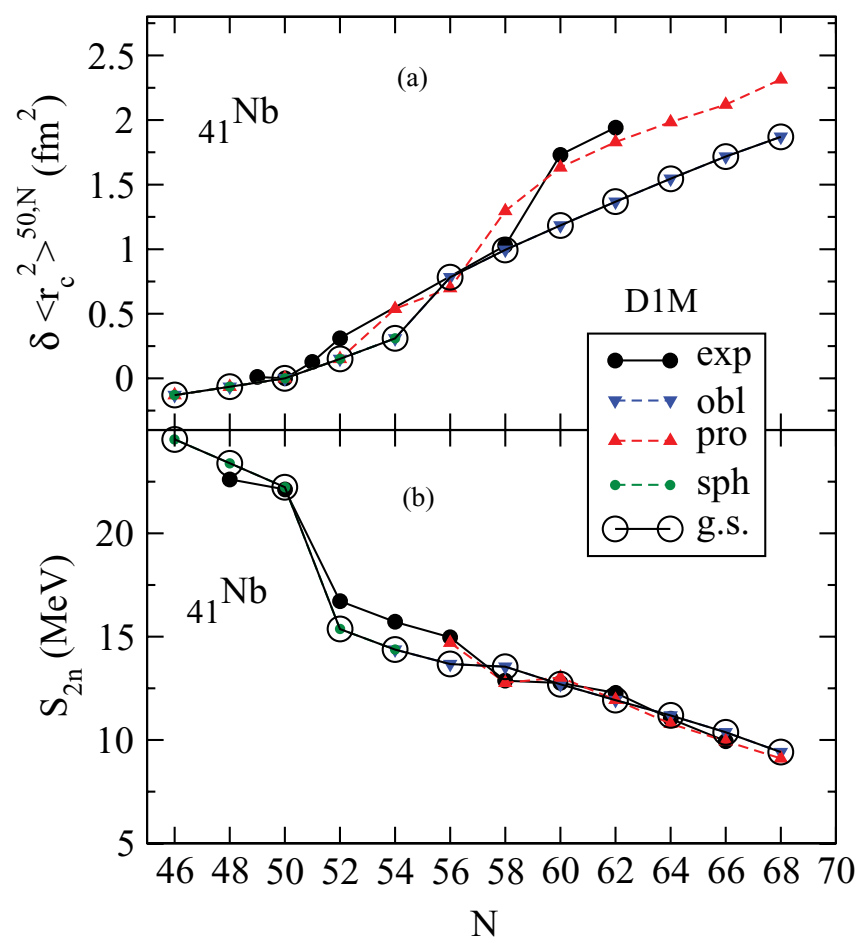

FIG. 12. (Color online) Same as in Fig. 9, but for niobium isotopes and Gogny D1M.

$N=58$ and $N=60$. As shown, the origin of the jump is related to the shape transition from slightly oblate shapes to well-deformed prolate configurations beyond $N=60$. The charge radius is again shown to be a nuclear property very sensitive to those shape variations. It is also worth mentioning that the sudden change of the radius is perfectly correlated with the change in the spin-parity of the ground states studied above.

In the case of $\mathrm{Nb}$ isotopes, shown in Figs. 11 and 12, the data are still scarce [33]. Nevertheless, a jump between $N=58$ and $N=60$ is also experimentally observed, although not as clearly as in the previous case. This is also associated with the transition from $9 / 2^{+}$to $\left(5 / 2^{+}\right)$observed in these isotopes between $N=58$ and $N=60$. As we have mentioned, the calculations produce only a gradual transition from spherical to oblate shapes, but the prolate shapes associated with the $5 / 2^{+}$states never become ground states. In order to follow the experimental trend we will need a transition at $N=60$ to get our calculations to the data points, which are explained in any case by the prolate shapes. In the past [14], similar problems were faced in the case of Mo $(Z=42)$ isotopes, which are the neighbors of $\mathrm{Nb}(Z=41)$ isotopes. In that case, it was demonstrated that for even-even Mo isotopes, the incipient emergence of triaxiality was at the origin of the experimentally observed radius evolution. The quadrupole deformations of the triaxial minima in Mo isotopes were very close to the prolate ones and therefore their effect on the radii were also similar to the prolate case. Similar arguments could be used here for $\mathrm{Nb}$ isotopes and one would expect the triaxial degrees of freedom to be more involved, but our present technical 
capability prevents us from carrying out triaxial calculations for odd- $A$ nuclei.

The results for $S_{2 n}$ agree in general with the measurements, but we get a systematic shell effect at $N=50$ stronger than that shown in the experiment. This is a well-known feature of any mean-field approach [43], which is resolved once configuration-mixing calculations beyond mean field, in the spirit of the generator coordinate method (GCM), are implemented in the formalism. In any case, it would be very helpful to extend and improve mass measurements to reduce the large uncertainties that still exist in neutron-rich isotopes. Most of the reported measurements in this exotic mass region are based on $\beta$-endpoint measurements, which are not completely reliable because they lead to very strong binding [31].

\section{CHARGE RADII SYSTEMATICS IN THE Kr-Mo REGION}

In this section we discuss the isotopic evolution of the charge radii in the whole region from $\operatorname{Kr}(Z=36)$ up to Mo $(Z=42)$. These isotope shifts have been found to be very sensitive probes of nuclear shape transitions and it is worth studying globally the whole Kr-Mo region and discussing the similarities and differences among the various isotopic chains.

In Fig. 13 we have compiled the measured isotope shifts $\delta\left\langle r_{c}^{2}\right\rangle$ for Mo [32], Nb [33], Zr [26], Y [30,36], Sr [22], Rb [21], and $\mathrm{Kr}[23]$ isotopes. These data are shown with solid symbols

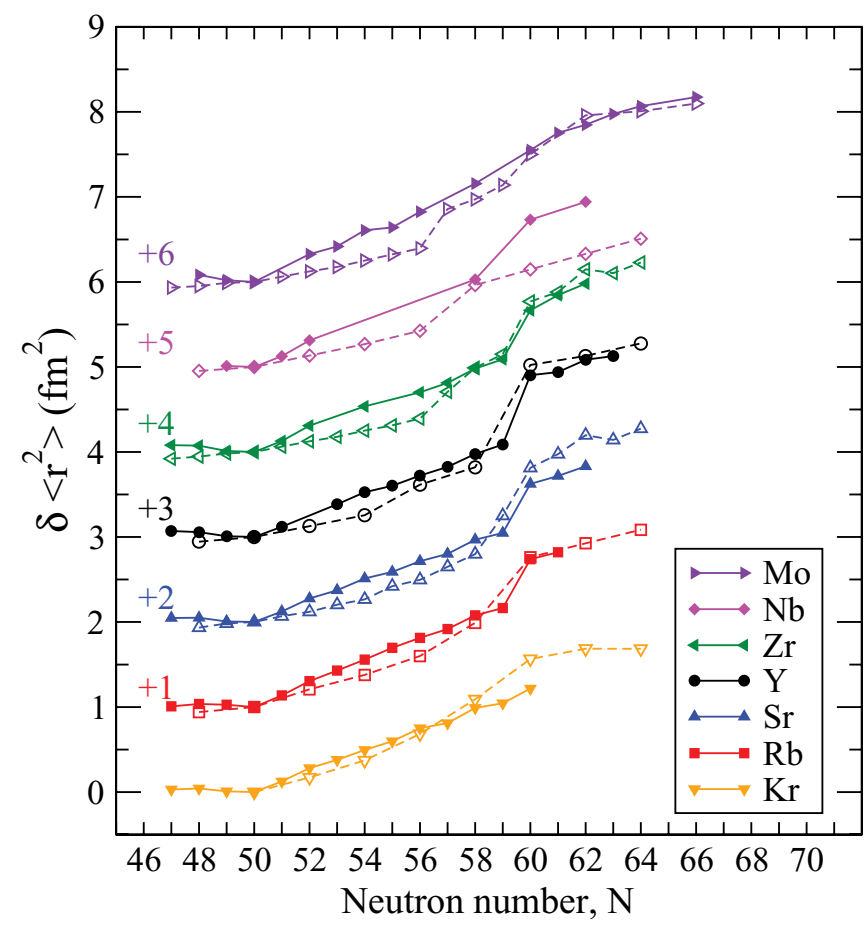

FIG. 13. (Color online) Gogny-D1S-HFB results for $\delta\left\langle r_{c}^{2}\right\rangle$ compared to the measured values for $\mathrm{Kr}, \mathrm{Rb}, \mathrm{Sr}, \mathrm{Y}, \mathrm{Zr}, \mathrm{Nb}$, and Mo isotopic chains. and connected with continuous lines. In addition, we also show our results from Gogny-D1S-HFB calculations, which are shown with open symbols connected with dashed lines. Very similar results are provided by the Gogny-D1M EDF. As shown in Fig. 13, this mass region is characterized by a jump of the mean-square charge radii at around $N=60$, which reflects the sudden increase of deformation or shape transition that occurs for these isotones. The increase in the charge radii is maximum for $\mathrm{Y}$ isotopes in the middle region and it corresponds to a change from oblate to prolate shapes at $N=60$, as shown in the previous section. Around $\mathrm{Y}$ nuclei, we find that $\mathrm{Zr}$ and $\mathrm{Sr}$ isotopes also show this large effect, which was studied in Refs. $[14,15]$ and interpreted as an oblate-prolate transition as well. The same is also true for $\mathrm{Rb}$ isotopes, as discussed in Ref. [16]. The behavior of Mo isotopes was studied in Refs. $[14,15]$, where it was shown that the suppression of the jump has its origin in the onset of triaxiality that smoothes the evolution of the radii. The case of $\mathrm{Nb}$ isotopes studied in this paper shows strong similarities with the case of Mo isotopes and triaxiality is again the likely reason for the discrepancies.

Finally, we also include results for $\mathrm{Kr}$ isotopes, measured in Ref. [23]. The isotope shifts for $\mathrm{Kr}$ isotopes do not show any abrupt change, but increase smoothly with the neutron number $N$. To understand this behavior in $\mathrm{Kr}$ nuclei, at variance with the abrupt change observed in the heavier neighboring isotopic chains, we have studied the evolution of the corresponding potential energy surfaces (PESs) (i.e., $Q-\gamma$ energy contour plots) in even-even $\mathrm{Kr}$ isotopes with the help of constrained HFB calculations along the lines discussed in Refs. [6,7]. We find that the lighter $\mathrm{Kr}$ isotopes present very shallow PESs centered at the spherical shapes. Heavier $\mathrm{Kr}$ nuclei gradually become oblate in their ground states with prolate configurations at higher energies. Therefore, the oblate configurations are stabilized in $\mathrm{Kr}$ isotopes and we do not find any transition to prolate shapes. The result is a smooth variation of the ground-state structure and, as a consequence, of the isotope shifts. To illustrate this point we show in Fig. 14 the triaxial landscapes for even-even $\mathrm{Kr}$ isotopes in the vicinity of $N=60$. We can see that the ground state in ${ }^{92} \mathrm{Kr}$ [Fig. 14(a)] is oblate with a very soft variation in $Q$ and especially in the $\gamma$ direction. The nucleus ${ }^{94} \mathrm{Kr}$ [Fig. 14(b)] develops an oblate ground state, which is again $\gamma$ soft. A prolate saddle point is also apparent. The heavier isotopes ${ }^{96} \mathrm{Kr}(N=60)$ (c) and ${ }^{98} \mathrm{Kr}(N=62)$ (d) develop two well-defined oblate and prolate minima, separated by energy barriers in the $Q$ and $\gamma$ degrees of freedom. The ground state always corresponds to the oblate shapes, while the prolate shapes appear about $1 \mathrm{MeV}$ higher in energy.

In general, our theoretical calculations successfully describe this phenomenology. The smooth behavior observed in $\mathrm{Kr}$ isotopes is a consequence of the stabilization of the oblate shapes along the isotopic chain. The sudden increase of the charge radii at $N=60$ observed in $\mathrm{Rb}, \mathrm{Sr}, \mathrm{Y}$, and $\mathrm{Zr}$ isotopes is explained by the oblate-to-prolate shape transition. Finally, the tendency to a smooth behavior observed again in $\mathrm{Nb}$ and Mo isotopes is explained by the onset of a region of triaxiality. 

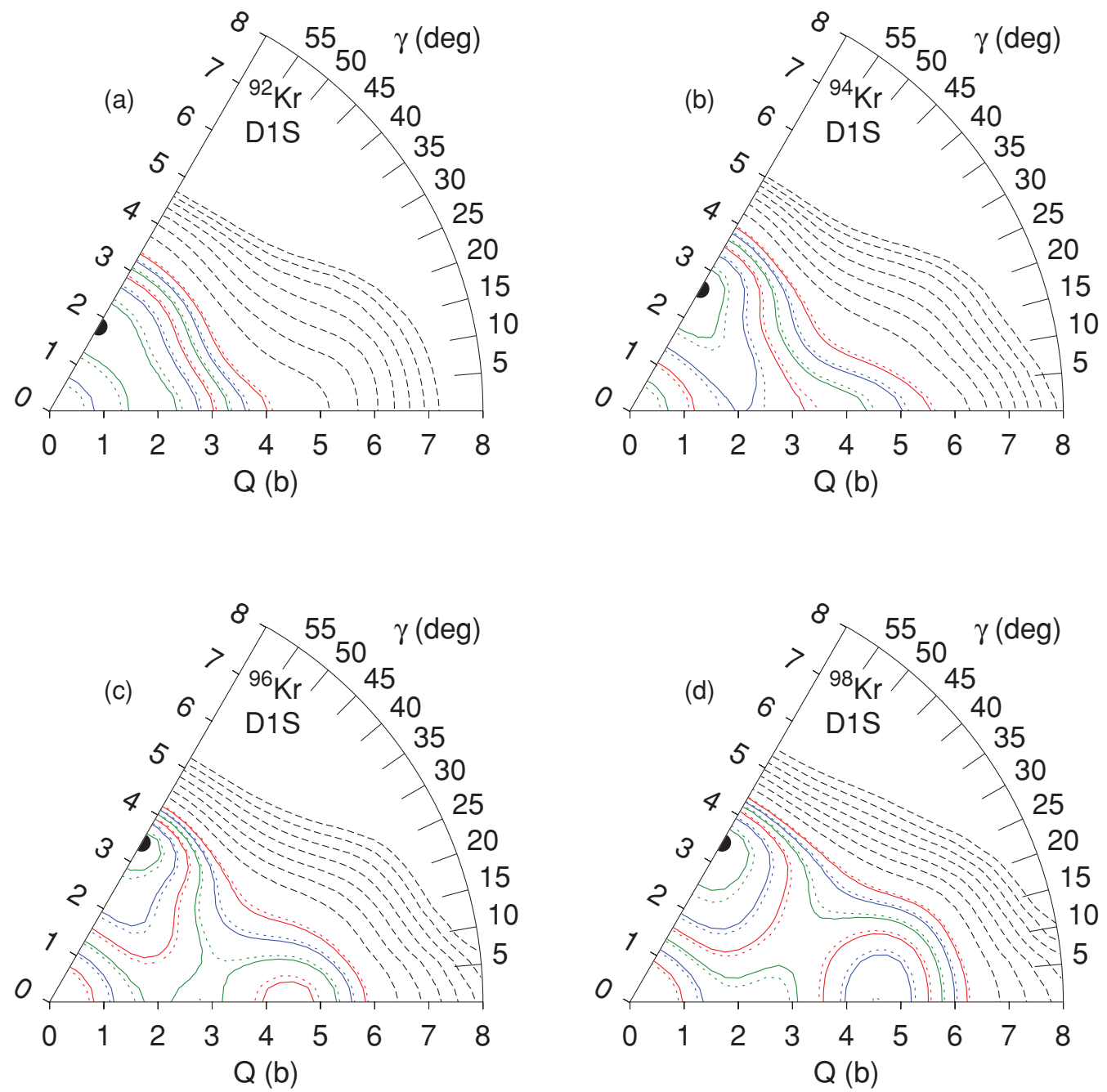

FIG. 14. (Color online) $Q-\gamma$ planes for ${ }^{92} \mathrm{Kr}$ (a), ${ }^{94} \mathrm{Kr}$ (b), ${ }^{96} \mathrm{Kr}$ (c), and ${ }^{98} \mathrm{Kr}$ (d) with the Gogny-D1S EDF. The absolute minimum is marked by a solid semicircle. The full contour lines correspond to energies $\epsilon_{C}$ (relative to the absolute minimum) of $0.25,0.75,1.25,1.75$, 2.25 , and $2.75 \mathrm{MeV}$. Close to those full-line contours, other contour lines corresponding to $\epsilon_{C}+0.1 \mathrm{MeV}$ are also depicted. The purpose of these dotted contour lines is to give the direction of increasing energy as well as a visual idea of the corresponding slope. Finally, the dashed contour lines corresponding to energies from 4 to $10 \mathrm{MeV}$ in steps of $1 \mathrm{MeV}$ are depicted to mark the region where the potential energy starts to grow rapidly.

\section{CONCLUSIONS}

In this work we have studied the shape evolution in odd- $A$ yttrium and niobium isotopes from microscopic self-consistent Gogny-EDF HFB-EFA calculations. We have also analyzed the isotopic evolution of one-quasiproton configurations, comparing the predictions of the D1S and D1M parametrizations of the Gogny EDF and demonstrating the robustness of our calculations.

Two-neutron separation energies, charge radii, and the spinparity of the ground states have been analyzed in a search for signatures of shape transitions. We have found that the charge radii and the spin-parity of the ground states are very sensitive to shape changes in these isotopes. In addition, the signatures found are all consistent with each other. We have also shown that the quality of the spectroscopic results obtained with the recent D1M incarnation of the Gogny EDF is comparable to the quality obtained with the standard D1S parametrization. We conclude that both D1M and D1S parametrizations reproduce, at least qualitatively, the main features observed in the isotopic trends of the considered neutron-rich isotopes.

The observed spin-parity of the ground states exhibits a sudden change between $N=58$ and $N=60$ isotopes in both isotopic chains. This is a clear signature of a nuclear structure transition, which is correlated to the observation of a similar change in the charge radii. According to our calculations, this structural change is explained by a shape transition taking place at this neutron number. The relevant feature to stress is that the jump in both spin-parity and charge radius is a signature of a shape transition, where the systematics are abruptly changed to a new situation. In the case of $\mathrm{Y}$ isotopes, the experimental spin-parities are well reproduced, except in the case of $N=58$, where the calculation predicts a transition going across an oblate configuration, which was 
not experimentally observed. In the case of $\mathrm{Nb}$ isotopes, the predicted transition is to oblate states, while the experimental spin-parities of heavier isotopes do not support these results. Since this is a region of an emergent triaxiality, as shown in neighboring even-even nuclei, this is a plausible explanation of the discrepancy. Finally, one should also note that the experimental spin-parity assignments in the heavier isotopes of both chains are either uncertain or taken from systematics, and therefore subject to revision.

We have performed a systematic study of the behavior of the charge radii in the region of neutron-rich isotopes from $\operatorname{Kr}(Z=36)$ up to Mo $(Z=42)$ to stress the manifest enhanced sensitivity of these observables to shape transitions. Comparison with the available data from laser spectroscopy demonstrates the quality and robustness of the Gogny-HFB description that is able to reproduce the main features of this behavior and offers an intuitive interpretation in terms of sharp or soft shape transitions, as well as triaxiality.

The experimental information available in neutron-rich $\mathrm{Y}$ and $\mathrm{Nb}$ isotopes, and in general in this mass region, is still incomplete. It would be desirable to extend the experimental programs for masses, charge radii, and spectroscopic measurements to these exotic regions at existing facilities such as the tandem Penning trap mass spectrometer (ISOLTRAP) [12] at the On-Line Isotope Mass Separator (ISOLDE) facility at CERN and the Ion Guide Isotope Separator On-Line (IGISOL) facility [11] at the University of Jyväskylä, or at future ones such as the precision measurements of very short-lived nuclei using an advanced trapping system for highly charged ions (MATS) and laser spectroscopy (LaSpec) [10] at the Facility for Antiproton and Ion Research (FAIR), where we can learn much about structural evolution in nuclear systems.
Although the present theoretical approach explains reasonably well the basic features observed in the data, theoretical efforts should still be pushed forward by improving the formalism to include triaxial degrees of freedom in odd- $A$ isotopes, in particular in those regions where this can be an issue, or by dealing with the odd systems with exact blocking treatments. The quality of our mean-field description could be also improved from configuration-mixing calculations in the spirit of the GCM with the quadrupole moment as the collective coordinate. It is already known [43] that such a configuration mixing reduces the jump in the predicted $S_{2 n}$, as compared to pure mean-field predictions, when crossing shell closures, thus improving the agreement with experiment. This could be particularly relevant for the light isotopes considered in the present study, where the spherical minima are rather shallow. For heavier isotopes, the two minima, oblate and prolate, are separated by spherical barriers of about $3 \mathrm{MeV}$ and typically appear about $1 \mathrm{MeV}$ apart. The effect here is not expected to be significant because a single shape would be enough to account for the properties studied.

\section{ACKNOWLEDGMENTS}

This work was supported by MICINN (Spain) under Grants No. FIS2008-01301, No. FPA2009-08958, and No. FIS2009-07277 and Consolider-Ingenio 2010 Programs CPAN CSD2007-00042 and MULTIDARK CSD2009-00064. R.R. acknowledges valuable suggestions from Professor J. Äystö, Professor I. Moore, and the experimental teams of the University of Jyväskylä, Finland.
[1] J. L. Wood, K. Heyde, W. Nazarewicz, M. Huyse, and P. Van Duppen, Phys. Rep. 215, 101 (1992).

[2] M. Bender, P.-H. Heenen, and P.-H. Reinhard, Rev. Mod. Phys. 75, 121 (2003).

[3] R. Rodríguez-Guzmán and P. Sarriguren, Phys. Rev. C 76, 064303 (2007).

[4] P. Sarriguren, R. Rodríguez-Guzmán, and L. M. Robledo, Phys. Rev. C 77, 064322 (2008).

[5] L. M. Robledo, R. R. Rodríguez-Guzmán, and P. Sarriguren, Phys. Rev. C 78, 034314 (2008).

[6] L. M. Robledo, R. Rodríguez-Guzmán, and P. Sarriguren, J. Phys. G 36, 115104 (2009).

[7] R. Rodríguez-Guzmán, P. Sarriguren, L. M. Robledo, and J. E. García-Ramos, Phys. Rev. C 81, 024310 (2010).

[8] B. Cheal and K. T. Flanagan, J. Phys. G 37, 113101 (2010).

[9] D. Lunney, J. M. Pearson, and C. Thibault, Rev. Mod. Phys. 75, 1021 (2003).

[10] D. Rodríguez et al., Eur. Phys. J. Spec. Top. 183, 1 (2010).

[11] A. Jokinen et al., Int. J. Mass Spectrom. 251, 204 (2006).

[12] M. Mukherjee et al., Eur. Phys. J. 35, 1 (2008).

[13] F. C. Charlwood et al., Hyperfine Interact. 196, 143 (2010).

[14] R. Rodríguez-Guzmán, P. Sarriguren, L. M. Robledo, and S. Perez-Martin, Phys. Lett. B 691, 202 (2010).

[15] R. Rodríguez-Guzmán, P. Sarriguren, and L. M. Robledo, Phys. Rev. C 82, 044318 (2010).
[16] R. Rodríguez-Guzmán, P. Sarriguren, and L. M. Robledo, Phys. Rev. C 82, 061302(R) (2010).

[17] P. Sarriguren and J. Pereira, Phys. Rev. C 81, 064314 (2010).

[18] A. Aprahamian, K. Langanke, and M. Wiescher, Prog. Part. Nucl. Phys. 54, 535 (2005).

[19] S. Goriely, N. Chamel, and J. M. Pearson, Phys. Rev. Lett. 102, 152503 (2009)

[20] J. J. Cowan, F.-K. Thielemann, and J. W. Truran, Phys. Rep. 208, 267 (1991).

[21] C. Thibault et al., Phys. Rev. C 23, 2720 (1981).

[22] F. Buchinger et al. Phys. Rev. C 41, 2883 (1990); 42, 2754 (1990).

[23] M. Keim et al., Nucl. Phys. A 586, 219 (1995).

[24] W. Urban et al., Nucl. Phys. A 689, 605 (2001).

[25] G. Lhersonneau, B. Pfeiffer, H. Gabelmann, and K.-L. Kratz, Phys. Rev. C 63, 054302 (2001).

[26] P. Campbell et al., Phys. Rev. Lett. 89, 082501 (2002).

[27] U. Hager et al., Phys. Rev. Lett. 96, 042504 (2006).

[28] D. Bucurescu et al., Phys. Rev. C 76, 064301 (2007).

[29] S. Rahaman et al., Eur. Phys. J. A 32, 87 (2007).

[30] B. Cheal et al., Phys. Lett. B 645, 133 (2007).

[31] U. Hager et al., Nucl. Phys. A 793, 20 (2007).

[32] F. C. Charlwood et al., Phys. Lett. B 674, 23 (2009).

[33] B. Cheal et al., Phys. Rev. Lett. 102, 222501 (2009).

[34] J. K. Hwang et al., Phys. Rev. C 80, 037304 (2009). 
[35] G. S. Simpson et al., Phys. Rev. C 82, 024302 (2010).

[36] K. Baczynska et al., J. Phys. G 37, 115103 (2010).

[37] J. Skalski, S. Mizutori, and W. Nazarewicz, Nucl. Phys. A 617, 281 (1997).

[38] F. R. Xu, P. M. Walker, and R. Wyss, Phys. Rev. C 65, 021303(R) (2002).

[39] P. Möller, J. R. Nix, W. D. Myers, and W. J. Swiatecki, At. Data Nucl. Data Tables 59, 185 (1995)

[40] P. Möller et al., At. Data Nucl. Data Tables 94, 758 (2008).

[41] G. A. Lalazissis, S. Raman, and P. Ring, At. Data Nucl. Data Tables 71, 1 (1999).

[42] M. Bender, G. F. Bertsch, and P.-H. Heenen, Phys. Rev. C 73, 034322 (2006).

[43] M. Bender, G. F. Bertsch, and P.-H. Heenen, Phys. Rev. C 78, 054312 (2008).

[44] S. Hilaire and M. Girod, Eur. Phys. J. A 33, 33 (2007).

[45] J.-P. Delaroche, M. Girod, J. Libert, H. Goutte, S. Hilaire, S. Péru, N. Pillet, and G. F. Bertsch, Phys. Rev. C 81, 014303 (2010).

[46] J. Dechargé and D. Gogny, Phys. Rev. C 21, 1568 (1980).

[47] T. Duguet, P. Bonche, P.-H. Heenen, and J. Meyer, Phys. Rev. C 65, 014310 (2001).

[48] L. Bonneau, P. Quentin, and P. Möller, Phys. Rev. C 76, 024320 (2007).

[49] S. Perez-Martin and L. M. Robledo, Phys. Rev. C 78, 014304 (2008).
[50] N. Schunck, J. Dobaczewski, J. McDonnell, J. More, W. Nazarewicz, J. Sarich, and M. V. Stoitsov, Phys. Rev. C 81, 024316 (2010).

[51] K. J. Pototzky et al., Eur. Phys. J. A 46, 299 (2010).

[52] J. F. Berger, M. Girod, and D. Gogny, Nucl. Phys. A 428, $23 \mathrm{c}$ (1984).

[53] J. L. Egido, L. M. Robledo, and R. R. Rodríguez-Guzmán, Phys. Rev. Lett. 93, 082502 (2004).

[54] G. F. Bertsch, M. Girod, S. Hilaire, J.-P. Delaroche, H. Goutte, and S. Péru, Phys. Rev. Lett. 99, 032502 (2007).

[55] S. Péru, J. F. Berger, and P. F. Bortignon, Eur. Phys. J. A 26, 25 (2005).

[56] S. Goriely, S. Hilaire, M. Girod, and S. Péru, Phys. Rev. Lett. 102, 242501 (2009).

[57] J. L. Egido, J. Lessing, V. Martin, and L. M. Robledo, Nucl. Phys. A 594, 70 (1995).

[58] P. Ring and P. Schuck, The Nuclear Many-Body Problem (Springer, Berlin, 1980).

[59] H. J. Mang, Phys. Rep. 18, 325 (1975).

[60] [http://www-phynu.cea.fr/science_en_ligne/carte_potentiels _microscopiques/carte_potentiel_nucleaire_eng.htm].

[61] Evaluated Nuclear Structure Data Files (ENSDF) [http://www.nndc.bnl.gov/ensdf].

[62] G. Audi, A. H. Wapstra, and C. Thibault, Nucl. Phys. A 729, 337 (2003). 